\title{
THE METHOD OF DETERMINATION OF HEAT EMISSION CHARACTERISTICS IN PISTON SELF-IGNITION INTERNAL COMBUSTION ENGINE
}

The article presents the method of plotting real indicator diagrams for piston internal combustion engines. Owing to the analysis of the diagrams, it is possible to determine the self-ignition delay period and also the characteristics of the relative amount of heat emitted in combustion process. The methodology worked out by the author accounts for changes in the amount and thermodynamic properties of the working medium present in the cylinder.

\section{Introduction}

Thermodynamic and thermochemical processes taking place in the cylinder of a piston, internal combustion engine have been thoroughly investigated, among others, in the USA, Japan and EU countries. The research is stimulated by the development of electronic systems controlling the engine work, which are widely applied to automotive vehicles. Those systems make the engine attain the required power and the optimum work indicators due to the minimum fuel consumption, the minimum emission of harmful components of exhaust gases and noise. They also provide for high reliability and long service life.

The combustion process is responsible for satisfying the abovementioned requirements. Therefore, it is the combustion process and possible improvements on it that draw the most attention. A cylinder pressure indicator diagram is useful here. It can be either computed or taken experimentally. With it, it is possible to determine and analyse the engine indicators and evaluate the characteristics of combustion heat emission. Moreover, it allows us to determine balance composition of basic components of combustion products versus the crankshaft rotation angle and also engine work hardness, etc.

In the paper, the author presents the basic issues concerning the analysis and simulation of indicator diagrams on the basis of randomly set characteristics of the relative amount of heat emitted in combustion.

\section{Experimental determination of an indicator diagram}

In order to determine the characteristics of the relative amount of heat emitted in combustion it is necessary to have a precise diagram illustrating pressure changes in the cylinder, instantaneous cylinder volumes, a reliable model of heat transfer between the working medium and combustion chamber walls. We also need to know the composition, amount and properties of the gas mixture.

The above-mentioned factors $[1,4,7,8]$ are decisive for the accuracy of heat emission characteristics determination. The error in the determination of those quantities may result from:

1) flow of lines of reference (environment),

2) thermal surge (shock),

3) error in specifying the piston position in TDC on an experimentally taken indicator diagram,

4) no averaging of repeatedly taken diagrams (averaging according to approx. $33 \div 50$ realisations) or smoothing of the averaged experimental indicator diagram,

5) quantification errors (sampling, resolution) in the real diagram.

The measurement system for investigations into piston internal combustion engines should also satisfy the following requirements:

- it must be equipped with accurate sensors,

- it should be possible to record a number of quantities simultaneously,

- measurements must be taken quickly and accurately, the values of measured quantities have to be recorded precisely,

- the quality control of measured quantities has to be kept while they are recorded and it must be possible to repeat a measurement if necessary.

The engine T359M investigations were carried out at the engine test stand equipped with eddy-current brake of WS-230 type manufactured by SCHENCK Company. The location of measurement points in engine indication is shown in Fig. 1.

Installed at the analyser input, replaceable insert NS-582 allows us to adjust the input signal voltage and to filter four simultaneously recorded and processed quantities presented in the digital form. The synchronous addressing system in the function of the crankshaft rotation angle makes it possible for the measured quan-

\footnotetext{
* Andrzej Ambrozik

Technical University of Kielce, Aleja Tysiąclecia Państwa Polskiego 7, PL-25314 Kielce, Poland, E-mail: silspal@eden.tu.kielce.pl
} 


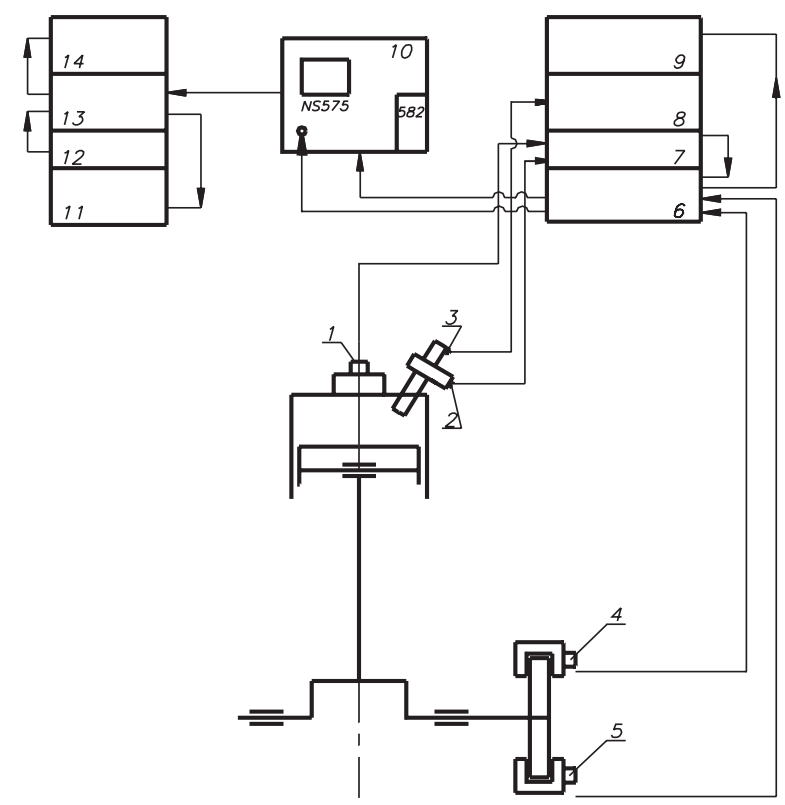

Fig. 1. Diagram of measurement points location in engine indication: 1 - piezoelectric sensor, type 642, of KISTLER company, for pressure measurements in the cylinder, 2 - piezoelectric sensor, type 1AE-123, manufactured by WRL, for fuel injection pressure measurements,

3 - capacitive needle lift sensor, 4 - photoelectric angular addressing sensor, 6 - angular addressing control system, 7 - charge amplifier, type MPU532, 8 - reactance transducer, type 51BO2, of DISA company, 9 - digital control voltmeter C-549A, 10 - digital recorder of measured runs, type NS-575, of NORTHERN company, with cartridge 582,

11 - printer DZM-180, 12 - disc memory UNIPOLBRIT, 13 - computer, 14 - monitor NEPTUN 156

tities to come as discrete 1024 data for $360^{\circ} \mathrm{CA}$ or $720^{\circ} \mathrm{CA}$. If one or two runs are recorded, the resolution can be increased twice or four times, respectively.

With the analyser reading system it is possible to:

- continuously watch the display of runs recorded in separate memory groups and also overlay them onto one another so that they can be compared,

- screen manually the registered data with a potentiometer, where data is read on the digital value indicator,

- reproduce the registered runs on $X-Y$ or $Y-t$ recorders, oscilloscopes or loop oscillographs,

- the system series or digital input is compatible with teleprinter, printer, cassette memory or digital monitor,

- the system parallel or digital output is compatible with printer.

The correctness of TDC setting in the required channel is checked with reference to the zero value of the first derivative of compression pressure changes run.

Measurements must be preceded by static and dynamic sensor calibration with respect to the measurement current conditions.

\section{The preparation of experimental an indicator diagrams for analysis}

If we want to prepare an experimentally taken diagram for further analysis, first of all, it is necessary to correctly specify the piston position in TDC on the indicator diagram and also to smooth the values of pressures. It should be remembered that the diagram of pressure in the working medium compression - expansion in the cylinder is not symmetrical with TDC, even if the combustion process does not take place. That results from the fact that there are the previous cycle exhaust gases leftovers and there is heat transfer between the working medium and the cylinder. Nevertheless, it often happens that in order to determine the piston TDC in the indicator diagram, we make use of the point, at which the first derivative of the compression pressure diagram equals zero.

For the sake of correction of TDC point location in the indicator diagram, it is also possible to rely on the condition that the mean indicated pressure pi, computed on the basis of measured pressure values equals the mean indicated pressure determined as the sum of exactly measured effective pressure and accurately determined mean pressure of mechanical losses occurring in the cylinder.

The correction of TDC point location in the indicator diagram can be also made on the basis of the condition that a fuel portion is completely burnt as a whole until the instant at which the outlet valve opens. This condition is satisfied when the relative amount of the heat emitted $x \approx 1,0$. The condition, however, is not completely true as it does not take into account the dissociation heat of such exhaust gases components as $\mathrm{H}_{2} \mathrm{O}$ and $\mathrm{CO}_{2}$, which can be quite large if the exhaust gases temperature exceeds $2500 \mathrm{~K}$.

Smoothing pressures in the indicator diagram is aimed at filtering out high frequency, often random diagram disturbances [8]. That involves connecting the measurement points with a smooth, interpolation curve. As a result, we obtain more regular characteristics of the relative amount of the heat emitted in a combustion process.

\section{Combustion incipience, self-ignition delay period}

Combustion process incipience in forced ignition engines is the time instant corresponding to the spark-over. Combustion incipience in self-ignition engines is connected with the fuel injection angle of advance and self-ignition delay period.

Self-ignition delay period is the time calculated from the beginning of the fuel injection into the combustion chamber to the chain-thermal explosion of the pre-flame reaction. It is registered in the indicator diagram as the start of very rapid increase in the working medium pressure and temperature caused by fuel combustion [3]. The length of this period significantly affects the initial stage of combustion: combustion rate, increase in pressure and temperature, engine starting properties, noise and others. N. N. Semenov, who investigated the kinetics of the pre-flame chain 
reaction and experimental data [7], stated that for the delay period $\tau_{i}$ of gas mixtures self-ignition, the following condition is satisfied: $\varphi \cdot \tau_{i}=$ const, where $\varphi$ is the factor of the self-acceleration of preflame reactions.

$$
\varphi=\varphi_{o} \mathrm{p}^{n} \exp \left(-\frac{E_{a}}{\bar{R}_{T}}\right)
$$

It is assumed that the constant rate of branching of the chain reaction $\varphi_{o}$ is proportional to the number of particle collisions, and the latter, in turn, depends on the fuel concentration in combustible mixture, that is on its pressure and temperature. This assumption and generalisation of the experimental data led A.I. Tolstov to the formula for the computation of self-ignition delay period:

$$
\tau_{i}=10^{-2} B\left(\frac{T_{p w}}{p_{p w}}\right)^{0.5} e^{\frac{E_{i}}{\bar{R}_{T}}}
$$

where: $B=B_{o}\left(1-K_{n}\right) ; B_{o} \approx 3.8 \cdot 10^{-4}$; whereas $K=1.6 \cdot 10^{-4}$ is a coefficient which accounts for the impact of the intensity of the working medium motion in the cylinder (proportional to the angular velocity $n$ ) on heat and mass transfer processes in the selfignition focus and other working medium parameters at the start of fuel injection. Quantity $E_{i}$ stands here for conventional energy of activation of pre-flame reactions and is a universal gas constant.

Formula (2) is widely applied to calculations of combustion in self-ignition engines. In order to enhance the accuracy and reliability of computation results, it is advisable to make the quantities $B_{o}$ or $E_{i}$ more precise in accordance with experimental data for a given engine.

Calculating quantity $\tau_{i}$ in accordance with the experimental indicator diagram, we take into account that a pressure changes rate at the end of compression is usually quite high and it is difficult to make up for errors while specifying the inflection point of a pressure $p$ curve, connected with combustion incipience. This point can be quite clearly determined with reference to the intersection point of temperature curves [6].

In order to do that, in accordance with values set $\mathrm{p}(\alpha)$, in the vicinity to the predicted point of self-ignition occurrence, we compute the set of temperatures from the state equation. We plot graphs $T(\alpha)$ and on their basis we determine the instant of self-ignition occurrence as the point of intersection of different characteristics $T(\alpha)$, that is: characteristics $T(\alpha)$ determined for compression and combustion. When we compute heat emission characteristics with the use of experimental indicator diagrams, the instant of self-ignition is determined with reference to the rapid increase in heat emission.

The methods of determining the instant of self-ignition occurrence are presented in the graphic form in Fig. 2. The intersection of characteristic $T(\alpha)$ for compression, determined at the assumption that air is the working medium with characteristic $T(\alpha)$, determined for $\alpha \leq \alpha_{T D C}$ at the assumption that the working medium are the products of complete and total combustion of the fuel fed to the engine. Curve $T(\alpha)$ for compression is plotted for $\alpha \geq \alpha_{p w}$, whereas curve $T(\alpha)$ for combustion - when $\alpha \leq \alpha_{T D C}$.

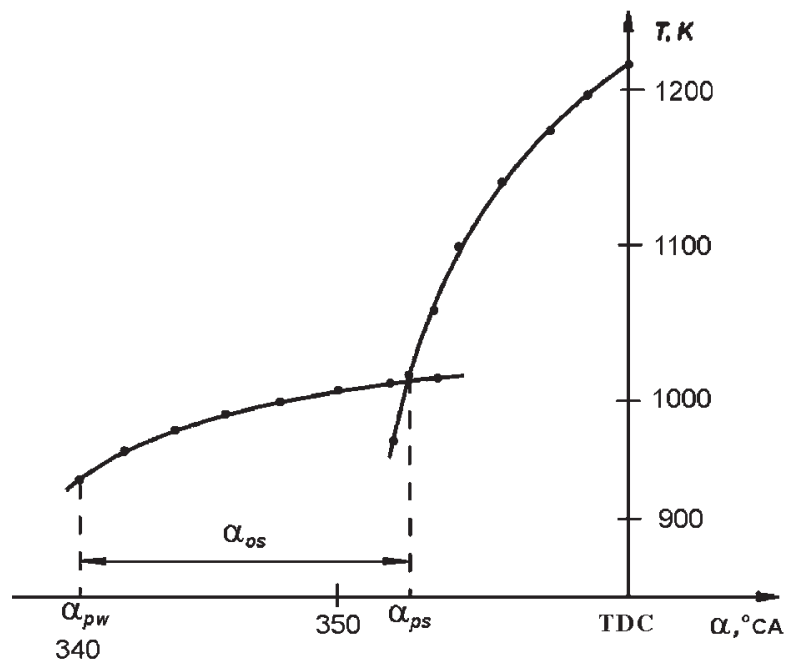

Fig. 2. The method of determination of the self-ignition delay period presented in the graphic form $\alpha_{p w}$ - fuel injection start, $\alpha_{p s}$ - combustion incipience

The self-ignition delay period is:

$$
\begin{aligned}
\alpha_{i} & =\alpha_{p s}-\alpha_{p w},{ }^{\circ} \mathrm{CA} \\
\tau_{i} & =\frac{\alpha_{i}}{6 n}, \mathrm{~s}
\end{aligned}
$$

The maximum rate of pressure increase in the self-ignition delay period is:

$$
\left(\frac{\Delta P}{\Delta \alpha}\right)_{\max }=\frac{p_{\alpha_{p s}}-p_{\alpha_{p w}}}{\alpha_{i}}, \frac{p a}{{ }^{\circ} C A}
$$

The conventional energy of activation of pre-flame reactions is determined from the dependence obtained after the logarithmisation of Tolstov's formula (2):

$$
E_{i}=\bar{R} T_{p w} \ln \frac{100 \tau_{i}}{B\left(\frac{T_{p w}}{p_{p w}}\right)^{0.5}} ;
$$

If the computed value of quantity $E_{i}$ is found in the interval $<23000,24000 \frac{\mathrm{J}}{\mathrm{mol}}>$, for the fuel fed to the self-ignition engine, it means that the determined value of fuel activation energy is sufficiently reliable. The value of activation energy determined in this way can be used in calculations of self-ignition delay period in the same engine fed with the same fuel but working under different conditions.

\section{The determination of characteristics of heat emission in combustion on the basis of an experimentally taken indicator diagram}

Having prepared an experimentally taken indicator diagram for further analysis we should compute: 
1. The amount of fuel charge burnt in a single working cycle of the engine:

$g_{c}=\frac{G e}{30 \cdot n \cdot z}$ or $g_{c}=\frac{p_{\alpha} V_{s} \eta_{v}}{\bar{R} T_{d} M_{o} \lambda}, \frac{k g}{\text { working cycle }}$

2. The value of the theoretical number of air kilo-moles necessary for complete and entire combustion of $1 \mathrm{~kg}$ of fuel of known elementary composition.

$M_{o}=\frac{1}{0,21}\left(\frac{C}{12}+\frac{H}{4}-\frac{O}{32}\right), \frac{\mathrm{kmol}}{\mathrm{kg} \cdot \text { fuel }}$

where: $C, H, O$ - carbon, hydrogen and oxygen mass participation in the fuel: $C+H+O=1$.

3. Number of kilo-moles of working medium performing a single working cycle of the engine:

$M_{c z}=\eta_{v} \frac{P_{d} V_{s}}{R T_{d}} \quad$ lub $\quad M_{c z}=\lambda g_{c} M_{o}$

where: $\eta_{v}$ - degree of cylinder filling; $\lambda$ - coefficient of excess air.

4. Kilomolar specific heats of the working medium.

The value of kilomolar specific heat is assumed to be linearly dependent on temperature [5], i.e.:

$c_{v}=a+b T_{s r} ; \kappa-1=\frac{\bar{R}}{\bar{c}_{v}}$

where: $\kappa$ - adiabate exponent; $a$ and $b$ - temperature coefficients of specific heats.

In the paper the following notation is assumed: coefficients for air $-a_{\lambda}$ and $b_{\lambda}$, those for exhaust gases $-a_{\gamma}$ and $b_{\gamma}$, and those for compression $-a_{s p r}$ and $b_{s p r}$.

5. Kilomolar specific heats of the working medium which changes its composition in combustion.

The values of these specific heats are computed from the dependence:

$\bar{c}_{v s p l}=a_{i}+b_{i} T_{i} ; \bar{c}_{\text {pspal }}=R+a_{i}+b_{i} T_{i}$

where:

$a_{i}=a_{s p r}(1-x)+x \cdot a_{\gamma}$

$\left.b_{i}=b_{s p r}(1-x)+x \cdot b_{\gamma}\right\}$

Quantity $x$ stands for the relative amount of the fuel that has come into reaction until considered time instant, it is calculated from the dependence:

$x=\frac{Q_{s}(\alpha)}{\xi g_{c} W_{u}}$

6. The changing number of kilo-moles of working medium in combustion can be accounted for by the introduction of instantaneous value of molar changes coefficient [6]. The values of theoretical and current coefficient of molar changes in the theory of internal combustion engines are calculated from the dependence:

$\beta_{o}=1+\frac{\frac{H}{4}+\frac{0}{32}}{\lambda M_{o}}$ and $\beta=\frac{\beta_{o}+\gamma}{1+\gamma}$

The instantaneous value of the coefficient of kilomolar changes in combustion can be calculated from the formula:

$\beta_{x}=1+\frac{\beta_{o}-1}{1+\gamma} \cdot x$

The instantaneous number of kilomoles of the working medium in the cylinder can be computed from the dependence:

$$
M_{x}=\lambda g_{c} M_{o} \beta_{x}
$$

\section{The determination of characteristics of the relative amount of heat emitted in combustion}

The relative amount of heat emitted in combustion is determined on the basis of the equation of the first law of thermodynamics and the state equation of the working medium in the cylinder:

$$
\left.\begin{array}{l}
d Q x=d U+p d V+d Q_{s c}+d Q_{d y s} \\
p V=M \bar{R} T
\end{array}\right\}
$$

The instantaneous and total amount of heat released in combustion is calculated from the dependence:

$$
d Q_{x}=\xi g_{c} W_{u} d_{x} ; Q=\xi g_{c} W_{u}
$$

where: $\xi$ - coefficient of heat emission in combustion.

The amount of heat transferred to the walls of the combustion chamber is calculated in accordance with Newton's formula [2]:

$$
d Q_{s c}=\alpha_{g} F d t
$$

Quantity $\alpha_{g}$ is calculated on the basis of empirical dependences provided in the literature on the subject [2]. Quantity F stands for the surface transferring the heat, whereas $d t$ can be substituted with a finite quantity:

$$
\Delta t=\frac{\alpha_{1}-\alpha_{i-1}}{6 \cdot n}
$$

where: $\alpha$ - crankshaft rotation angle kąt, computed in ${ }^{\circ} \mathrm{CA}$.

In the computation methodology put forward by the author, it is assumed that dissociation phenomenon does not occur in combustion, i.e. $Q_{d y s}=0$.

Inserting dependences (18), (19) and (20) into (17) and applying transformations, we obtain the formula which accounts for the calculation of the relative amount of heat released in combustion: 


$$
\begin{aligned}
& X_{i}=\frac{1}{\xi g_{c} W_{o}}\left\{\frac { 1 } { \kappa - 1 } \left[\kappa p_{i}+\frac{p_{i-1}}{2}\left(V_{i}-V_{i-1}\right)+\right.\right. \\
& \left.\left.+\frac{V_{i}+V_{i-1}}{2}\left(p_{i}-p_{i-1}\right)\right]+\alpha g_{i} F_{i} \frac{\alpha_{i}-\alpha_{i-1}}{6 \cdot n}\right\}
\end{aligned}
$$

The rate at which the relative amount of heat is emitted in combustion is calculated from the dependence:

$$
\dot{x}_{i}=\frac{x_{i}-x_{i-1}}{\alpha_{i}-\alpha_{i-1}}
$$

Fig. 3 presents an exemplary diagram $\dot{x}, x_{i}$ and $x_{s c}$ as well as $x$, which results from the analysis of indicator diagram of the engine T359 working when: $n=1900 \mathrm{~min}^{-1}, g_{c}=7.16 \cdot 10^{-5} \mathrm{~kg} / \mathrm{cycle}$, $M e=460 \mathrm{Nm}$ and injection advance angle $\alpha_{w w}=18{ }^{\circ} \mathrm{CA}$.

\section{Conclusions}

Owing to the method of analysing the real indicator diagram for piston self-ignition internal combustion engine put forward in the paper, it is possible to determine self-ignition delay period and the characteristics of the relative amount of heat emitted in combustion. Thus it allows us to estimate the amount of fuel burnt on the basis of kinetic and diffuse mechanism of combustion reaction as well as the duration of these combustion phases in the engine cylinder.

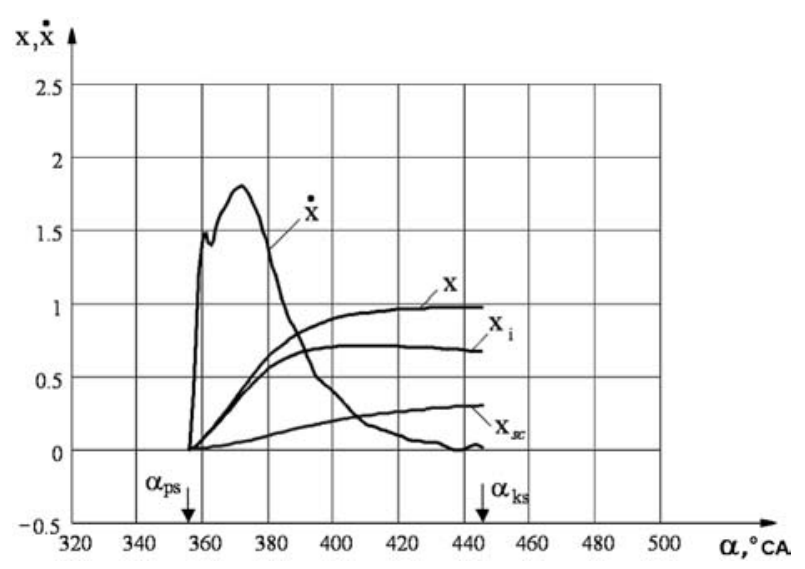

Fig. 3 Relative amounts of heat emitted in combustion and the rate $\dot{x}$, at which heat is emitted in engine T359 [3] working when: $n=1900 \mathrm{~min}^{-1}, g_{c}=7.16 \cdot 10^{-5} \mathrm{~kg} / \mathrm{cycle}, p_{p w}=18.5 \mathrm{MPa}$ and $\alpha_{w w}=18$ (CA ahead of TDC. $x$ - relative amount of emitted heat, $x_{i}$ - heat equivalent to indicated work, $x_{s c}$ - heat transferred out of combustion space walls, respectively. Quantities $\alpha_{p s}$ and ${ }_{k s}$ denote combustion incipience angle and combustion end angle, respectively.

The method worked out by the author could be applied to combustion process identification and also used to control the process course.

\section{References}

[1] AMBROZIK, A., SOBOCIŃSKI, R.: Analysis of Combustion Process in Piston Internal Combustion Engines on the Basis of Indicator Diagram (in Polish). ZN Transport no. 21, pp. 72-100, Warsaw, 1982.

[2] AMBROZIK A.: Empirical Dependence Classification for Heat Transfer Coefficient Determination in Piston Internal Combustion Engines (in Polish). Silniki spalinowe no. 4/19, ed. Zakłady Przemysłu Metalowego H. Cegielskiego, Poznan, 1987.

[3] AMBROZIK, A.: Evaluation Methods Improvement and Selection of Ways to Perfect the Perspective Indicators of Automotive Diesel Engines Made in Poland (in Russian). Habilitation Dissertation, Charkow, 1991.

[4] AMBROZIK, A., RAZUMOWSKI, M.: Model of Calculations for the Working Cycle of Piston Internal Combustion Engines (in Polish). Journal of KONES Internal Combustion Engines, Warsaw-Poznan, 1995.

[5] AMBROZIK, A., MARCENKO, A. P., PONIEWSKI, M., SZOKOTOW, N.K.: Exergy Analysis of Piston Internal Combustion Engines (in Polish). Ed. by the University of Technology, Kielce, 1998, p. 222.

[6] AMBROZIK, A., DANILCZYK, W., KRUCZYŃSKI, S.W.: Simulation of an indicator diagram of a diesel engine. Polish Academy of Sciences Branch in Lublin. Commission of Motorization and Power Engineering in Agriculture, vol. III, Lublin, 2003. pp. 12-17.

[7] SEMENOV, N. N.: Chain Relations (in Russian). L. Goschimizdat, 1934, p. 555.

[8] QANADILO, H.: Investigations into and Estimation of Heat Emission Characteristics in Piston, Self -Ignition Internal Combustion Engines (in Polish). Doctoral thesis. Warsaw, 2002.

[9] RYCHTER, T., TEODORCZYK, A.: Mathematical Modelling of Piston Engine Working Cycle (in Polish). PWN, Warsaw, 1990.

[10] SZCZĘSNY, P.: Numerical Modelling of Heat Emission Process in Internal Combustion Engine (in Polish). Doctoral thesis, Czestochowa University of Technology, Faculty of Machinery Design, Lublin, 1992. 